\title{
Macrophage activation by IFN-y triggers restriction of phagosomal copper from intracellular pathogens
}

\author{
Qian Shen, Matthew J. Beucler, Stephanie C. Ray, and Chad A. Rappleye \\ Department of Microbiology, Ohio State University, Columbus, OH 43210, USA
}

Objectives: This study investigates how macrophages alter copper availability to control intracellular pathogens and the mechanisms by which Histoplasma capsulatum combats copper restriction.

Methods: A forward genetic screen of $H$. capsulatum identified loci required for proliferation within macrophages. Quantitative RT-PCR (qRT-PCR) was used to measure the transcriptional response of Histoplasma yeasts to high or low copper. To estimate intraphagosomal copper concentrations in macrophages, a gfp transcriptional reporter was used to measure the activity of the copper-responsive CTR3 promoter. Histoplasma yeasts lacking Ctr3 were tested for virulence using a murine model of pulmonary histoplasmosis.

Results: The loss of the ability of Histoplasma yeast to replicate in macrophages was linked to disruption of the CTR3 locus, which encodes a fungal copper transporter. Loss of Ctr3 function impairs yeast growth in limiting copper consistent with Ctr3 functioning as a copper-specific importer. Transcription of the CTR3 gene is induced both by differentiation of $H$. capsulatum into pathogenic yeasts and by low available copper. Infection of macrophages with a strain having a transcriptional fusion to the CTR3 promoter demonstrated that phagosomes of non-activated macrophages have moderate copper levels that are not antimicrobial to $H$. capsulatum. However, IFN- $y$ activation of phagocytes causes phagosomal copper restriction both in culture and in vivo. Accordingly, in a respiratory model of histoplasmosis, Ctr3-deficient yeasts are fully virulent during innate immunity but are attenuated after the onset of adaptive immunity.

Conclusion: Activation of macrophages during cell-mediated immunity, switches the phagosome from a copper-replete to a copper-depleted environment, forcing $H$. capsulatum reliance on $\mathrm{Ctr} 3$ for copper acquisition.

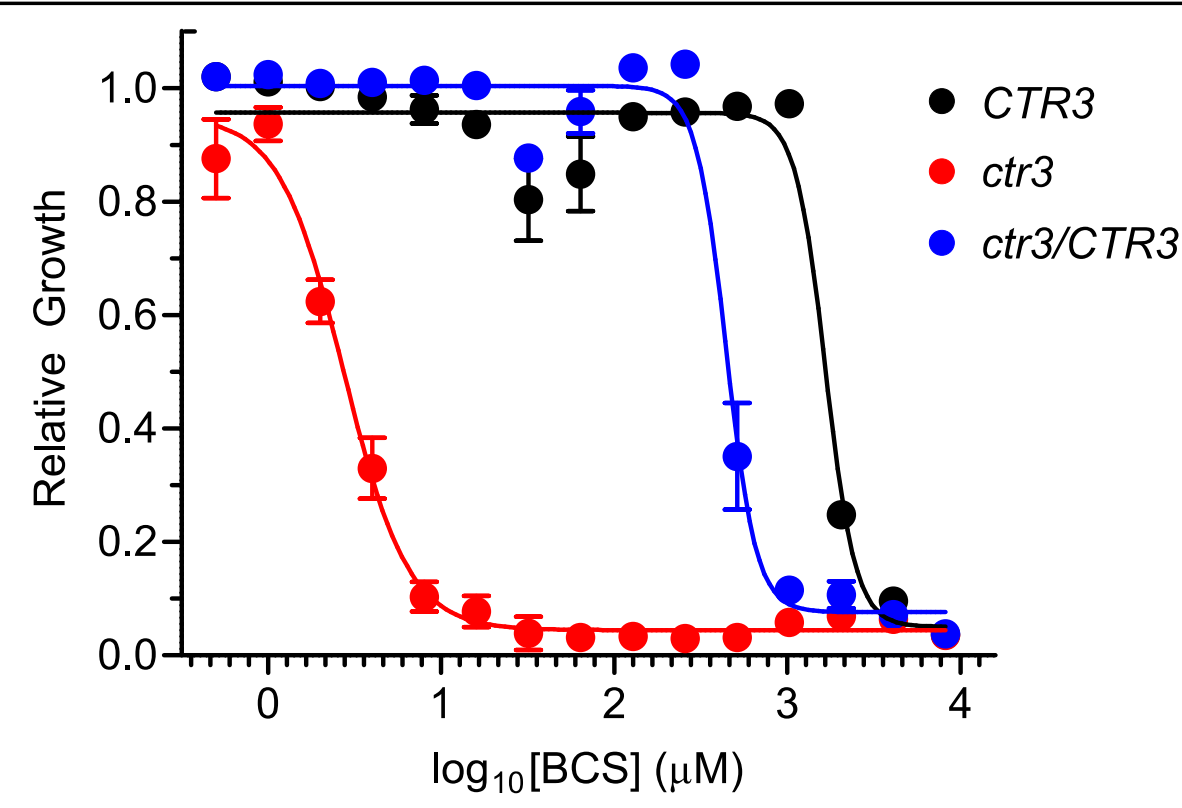

Figure 1. Ctr3 enables $\boldsymbol{H}$. capsulatum growth in limited copper. Dose response curves for Ctr3-expressing (CTR3, black), Ctr3-deficient (ctr3, red), and the complemented (ctr3/CTR3, blue) strains grown in liquid culture with the copper-specific chelator BCS.

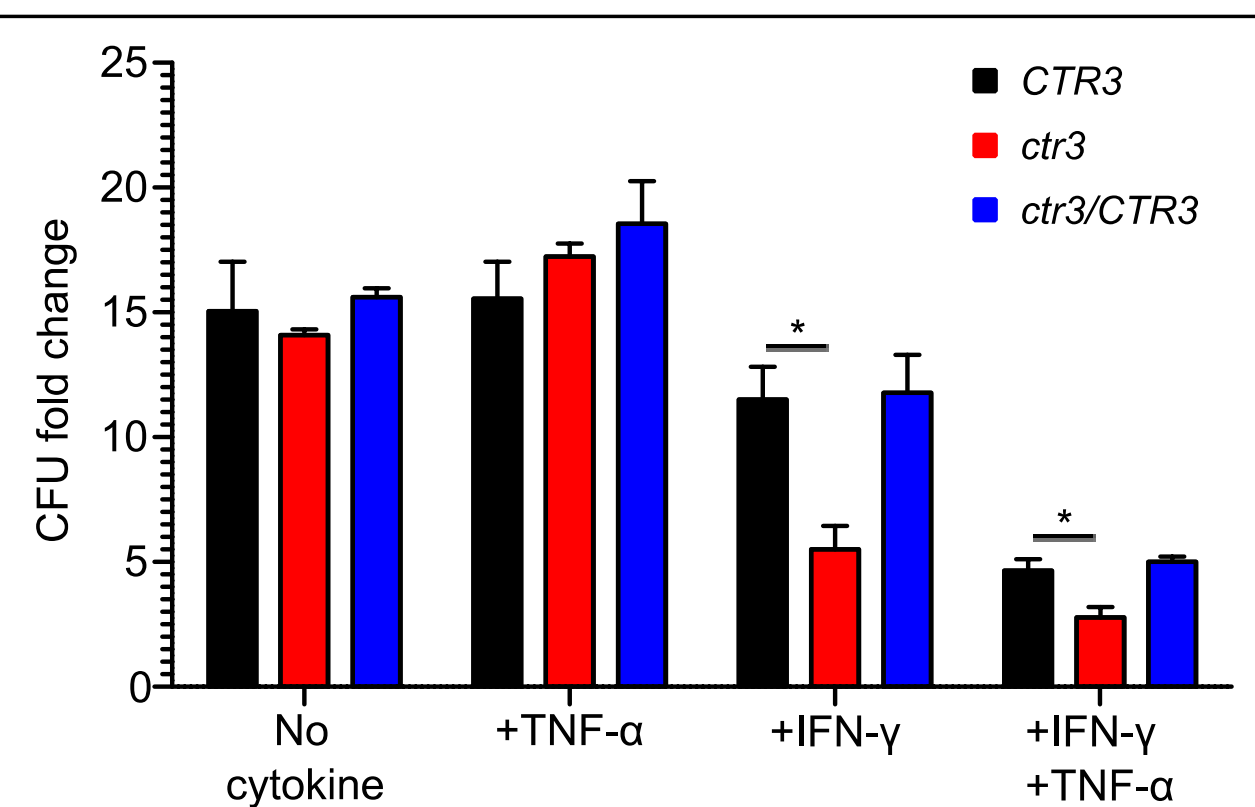

Figure 2. Activation of macrophages impairs proliferation of Ctr3deficient yeasts. Bone marrow derived macrophages (BMDMs) were activated with IFN- $\gamma$ and/or TNF- $\alpha$, and infected with Ctr3-expressing (CTR3, black), Ctr3-deficient (ctr3, red) and complemented (ctr3/CTR3, blue) H. capsulatum yeasts. Intracellular yeasts were enumerated (CFU) after 48 hours. Asterisks indicate significant differences $\left({ }^{*} P<0.05\right)$ in the intracellular proliferation of yeasts.

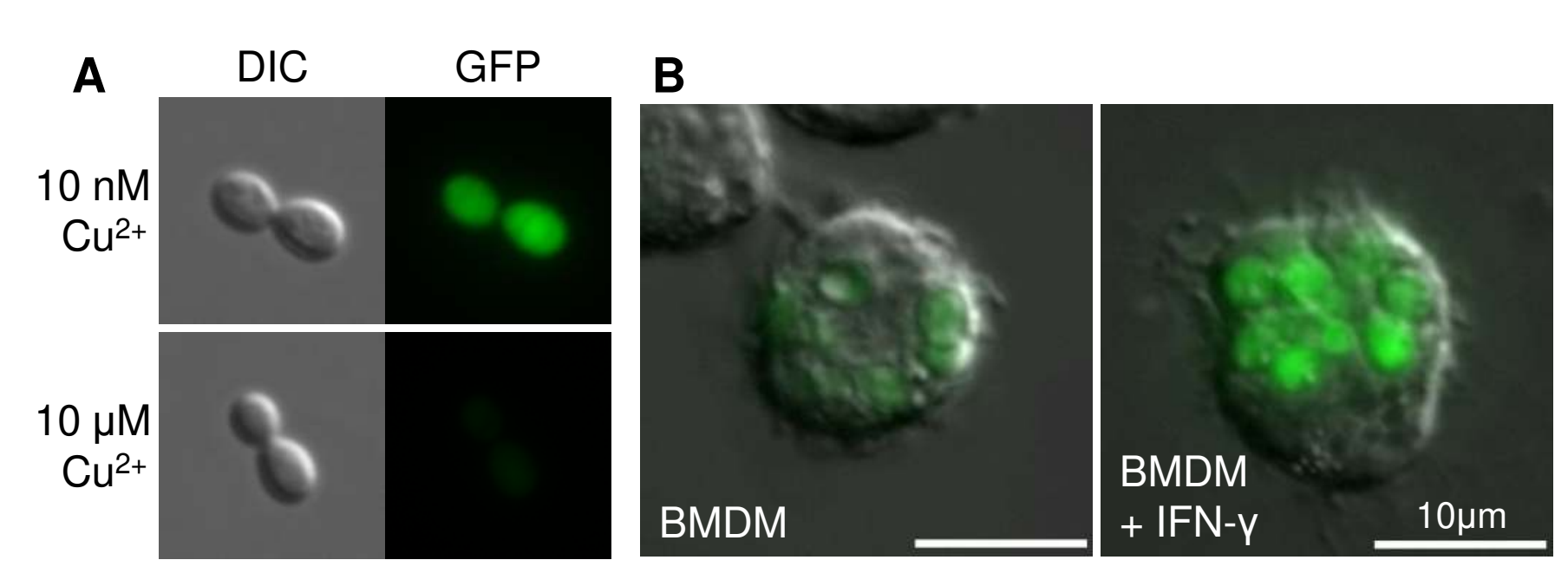

Figure 3. Activation of macrophages decreases phagosomal copper availability. (A) Representative images of $H$. capsulatum yeasts with the CTR3 promoter-gfp fusion grown in vitro with different copper concentrations. (B) CTR3 promoter activity in BMDMs with and without IFN-y activation as indicated by the fluorescence of the $\mathrm{P}_{\text {CTR3-grp }}$ transcriptional reporter fusion.

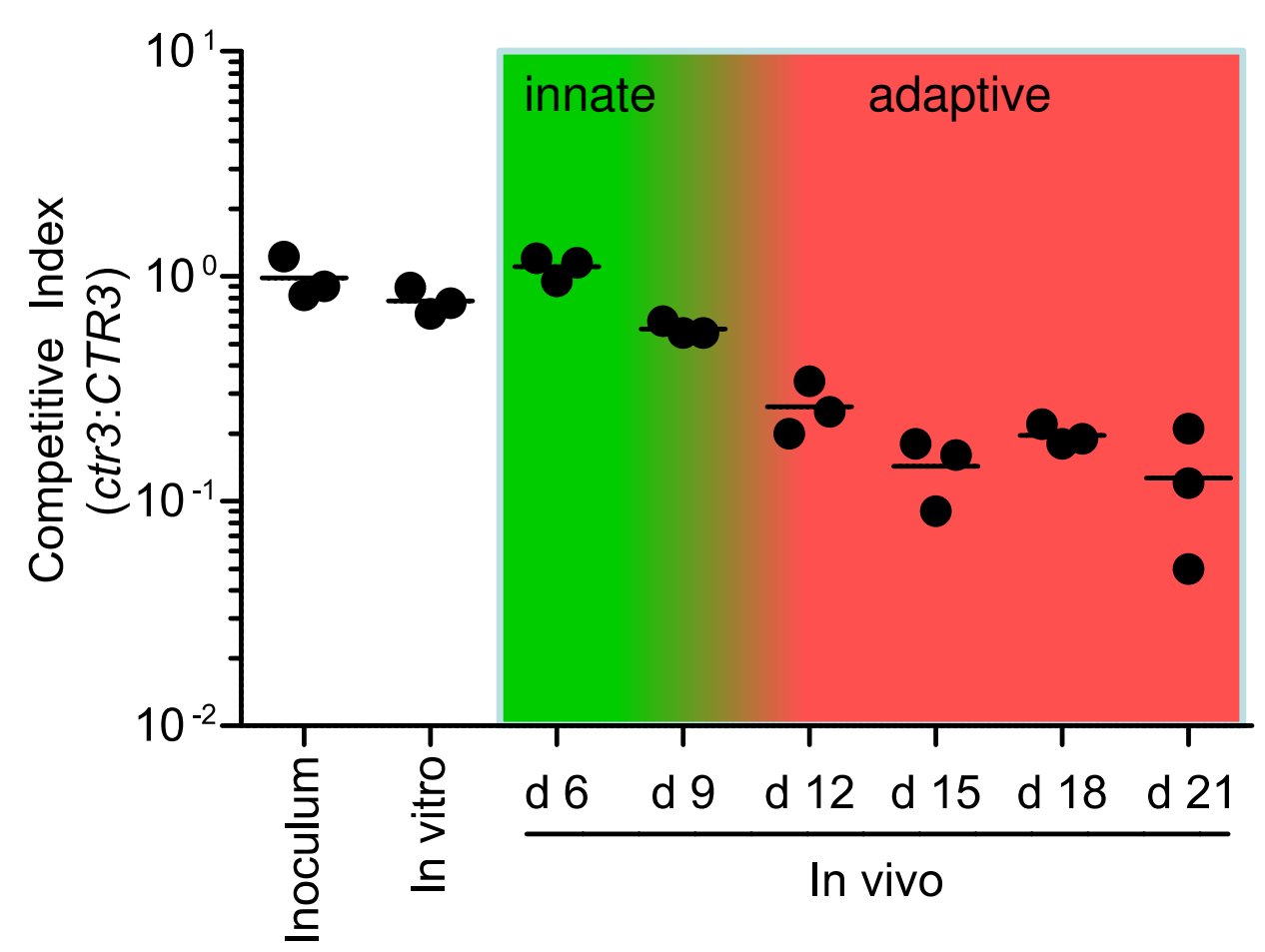

Figure 4. $H$. capsulatum virulence in vivo requires Ctr3 during the adaptive immune response. The relative fitness (competitive index) of Ctr3-deficient (ctr3) compared to Ctr3-producing (CTR3) yeasts was determined by co-infecting mice intranasally with equal amounts of CTR3 and ctr3 yeasts. Relative fungal burdens were determined for yeasts co-cultured in vitro and in the lungs at $6,9,12,15,18$, and 21 days post-infection. Asterisks indicate significant $\left({ }^{*} P<0.05,{ }^{* *} P<0.01\right)$ differences compared to the ctr3: CTR3 ratio of the inoculum.

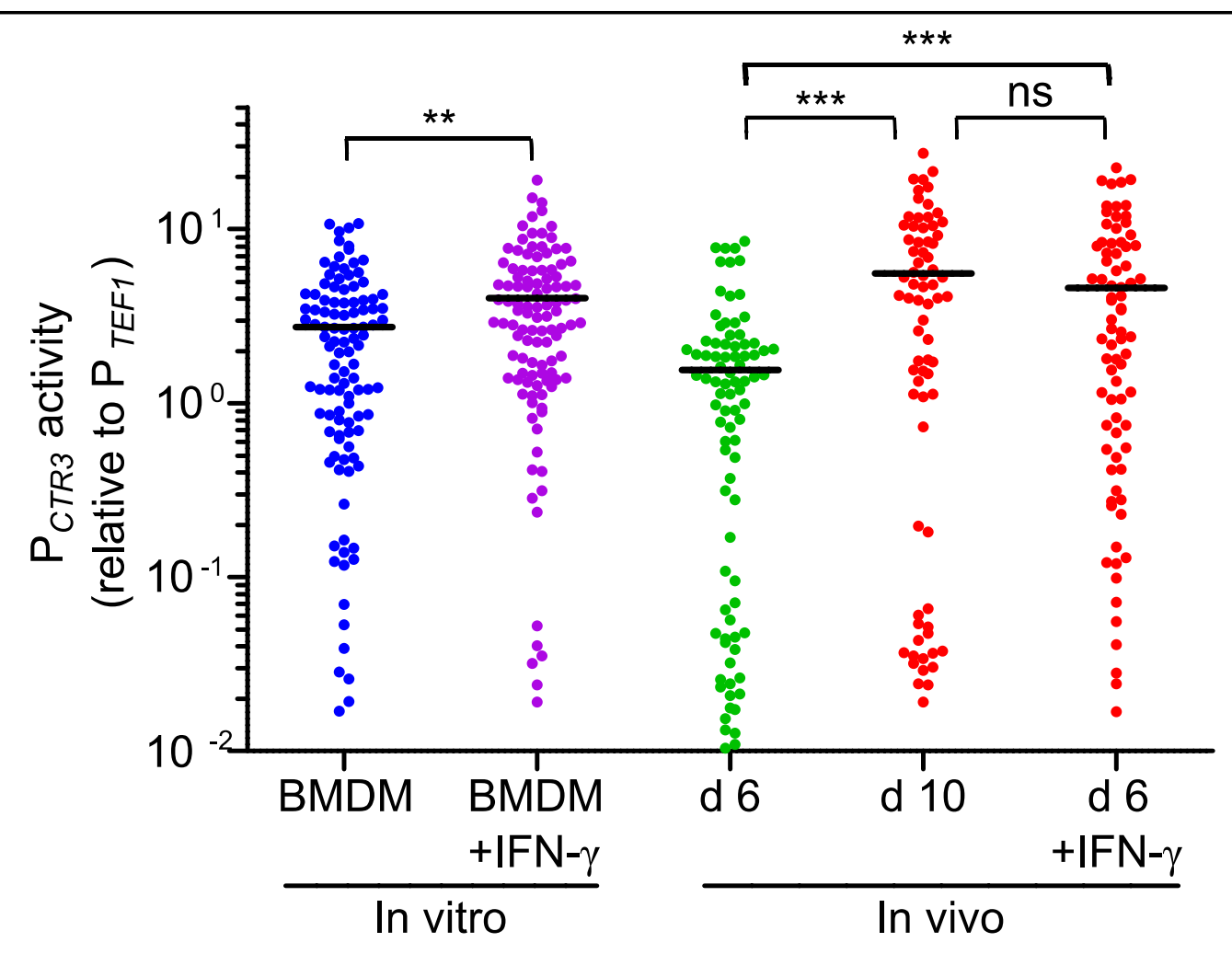

Figure 5. IFN- $\gamma$ treatment restricts copper availability in vivo. BMDMs or mice were infected with $H$. capsulatum yeast with TEF1 $\left(\mathrm{P}_{\text {TEF } 1}\right)$ or CTR3 $\left(\mathrm{P}_{\text {CTR3 }}\right)$ promoter-gfp fusions. CTR3 promoter activity yeasts following infection was determined by measurement of GFP-fluorescence by microscopy and normalized to the average TEF1 promoter activity. Data shows the distribution of CTR3 promoter activity and horizontal bars represent the mean CTR3 promoter activity. Asterisks indicate significant differences $\left({ }^{* *} P<0.01,{ }^{* * *} P<0.001\right)$. 